\title{
Investigation of Inconsistent ENDF/B-VII.1 Independent and Cumulative Fission Product Yields with Proposed Revisions
}

\author{
M.T. Pigni, ${ }^{1, *}$ M.W. Francis, ${ }^{1}$ and I.C. Gauld ${ }^{1}$ \\ ${ }^{1}$ Oak Ridge National Laboratory, Oak Ridge, TN 37831-6170, USA
}

(Received 29 May 2014; revised received 12 July 2014; accepted 16 July 2014)

\begin{abstract}
A recent implementation of ENDF/B-VII.1 independent fission product yields and nuclear decay data identified inconsistencies in the data caused by the use of updated nuclear schemes in the decay sub-library that are not reflected in legacy fission product yield data. Recent changes in the decay data sub-library, particularly the delayed neutron branching fractions, result in calculated fission product concentrations that do not agree with the cumulative fission yields in the library as well as with experimental measurements. To address these issues, a comprehensive set of independent fission product yields was generated for thermal and fission spectrum neutron-induced fission for ${ }^{235,238} \mathrm{U}$ and ${ }^{239,241} \mathrm{Pu}$ in order to provide a preliminary assessment of the updated fission product yield data consistency. These updated independent fission product yields were utilized in the ORIGEN code to compare the calculated fission product inventories with experimentally measured inventories, with particular attention given to the noble gases. Another important outcome of this work is the development of fission product yield covariance data necessary for fission product uncertainty quantification. The evaluation methodology combines a sequential Bayesian method to guarantee consistency between independent and cumulative yields along with the physical constraints on the independent yields. This work was motivated to improve the performance of the ENDF/B-VII.1 library for stable and long-lived fission products. The revised fission product yields and the new covariance data are proposed as a revision to the fission yield data currently in ENDF/B-VII.1.
\end{abstract}

\section{INTRODUCTION}

Fission product yields (FPYs) define the distributions of fission products following fission and depend on both the fissioning nuclide and the energy of the neutron causing fission. Accurate FPYs are required for a broad range of nuclear energy applications including reactor and fuel cycle safety analysis, spent nuclear fuel storage, transportation, and geological disposal analyses, and for applications including nuclear safeguards, non proliferation research, and nuclear forensic analysis [1. The most recent revision of nuclear data from the U.S. Nuclear Data Program is ENDF/B-VII.1 [2], released in 2011.

The ENDF/B-VII.1 fission product yield sub-library, with the exception of a new FPY evaluation for ${ }^{239} \mathrm{Pu}[3$, is based on the compilations developed by England and

\footnotetext{
* Corresponding author: pignimt@ornl.gov

This manuscript has been authored by the Oak Ridge National Laboratory, managed by UT-Battelle LLC under Contract No. DE-AC05-00OR22725 with the U.S. Department of Energy. The United States Government retains and the publisher, by accepting the article for publication, acknowledges that the United States Government retains a non exclusive, paid-up, irrevocable, world wide license to publish or reproduce the published form of this manuscript, or allow others to do so, for United States Government purposes.
}

Rider released in 1994 4 (see Tab. I). Namely, England and Rider produced a recommended set of independent and cumulative FPYs based on a compiled list of open literature measurements and calculated charge distributions. This set was the basis for the FPY evaluations used in ENDF/B-VI library released in 1993 and, subsequently, adopted by ENDF/B-VII.0 and -VII.1 libraries released in 2006 and 2011, respectively. The library in-

TABLE I. FPY evaluations in the ENDF-B/VII.1 library.

\begin{tabular}{r|r|c|l}
\hline \hline$\#$ & Nuclei & Year(s) & Author(s) \\
\hline 1 & ${ }^{227,229,232} \mathrm{Th}$ & 1994 & Ref. [4] \\
2 & ${ }^{231} \mathrm{~Pa}$ & 1994 & Ref. [4] \\
3 & ${ }^{232-238} \mathrm{U}$ & 1994 & Ref. [4] \\
4 & ${ }^{237,238} \mathrm{~Np}$ & 1994 & Ref. [4] \\
5 & ${ }^{238-242} \mathrm{Pu}$ & 1994,2011 & Refs. [4, [2] \\
6 & ${ }^{241,242 \mathrm{~m}, 243 \mathrm{Am}}$ & 1994 & Ref. [4] \\
7 & ${ }^{242-248} \mathrm{Cm}$ & 1994 & Ref. [4] \\
8 & ${ }^{249,251} \mathrm{Cf}$ & 1994 & Ref. [4] \\
9 & ${ }^{254} \mathrm{Es}$ & 1994 & Ref. [4] \\
10 & ${ }^{255} \mathrm{Fm}$ & 1994 & Ref. [4] \\
\hline \hline
\end{tabular}


cludes evaluations of both the independent and cumulative fission yields. An isotope's independent yield is the fraction of that nuclide produced directly from the fission process after emission of prompt neutrons but prior to any radioactive decays (including delayed neutron emission). Infact, the fission fragments are initially formed in highly excited states of neutron-rich nuclei and they release an average of about 2-3 prompt neutrons in order to reduce the neutron excess along with prompt $\gamma$-rays to reduce the energy excess. After these prompt decays, the cumulative yields, by contrast, are produced by slower decays $\left(\tau_{1 / 2}>1 \mathrm{~ms}\right)$, mostly, such as $\beta$-, $\alpha$-decay and delayed neutron emission. Therefore, cumulative yields equal the sum of all the independent yields for all decay precursors and they are inextricably linked to the independent yields by the decay schemes.

Since the initial release of the England and Rider yield data, improved nuclear decay schemes have been developed and are adopted in ENDF/B-VII.0 and -VII.1 decay sub-libraries. However, the new decay data can create internal inconsistencies with the legacy FPYs due to differences between the decay data used in the fission yield evaluation and current data. Changes in the decay schemes when used with the independent fission yields for fission product generation can result in calculated concentrations for the stable and long-lived nuclides at the end of the decay chains that do not agree with the cumulative yields in the evaluation. Because the cumulative yields for stable and longer-lived fission products are frequently determined accurately from experiment, it is essential that independent yields and decay data used by most isotope irradiation and decay simulation codes can reproduce the cumulative yields in the evaluations. Although such data inconsistencies are recognized within the nuclear data community, the practical implications on many applications are not widely known within the user community.

The data inconsistencies became evident in recent applications of the ORIGEN code when fission product noble gases were compared to measured xenon and krypton isotopes. Maintained as the depletion analysis module for the SCALE code [5], the current version of ORIGEN includes independent FPY data from England and Rider's compilations and nuclear decay data from ENDF/BVII.1. Calculated isotopic concentrations, particularly, of longer lived and stable isotopes using these data were found to have discrepancies compared to available experimental data and were inconsistent with the cumulative fission yields of England and Rider. These differences are the result of changes, primarily, in the delayed neutron $(\beta-\mathrm{n})$ branching fraction values used by England and Rider from those ones found in ENDF/B-VII.1 decay data. These changes introduce errors in the isotopic distribution of some stable and long-lived fission products of up to $5 \%$ in some cases compared to values predicted on the basis of the cumulative yields.

Motivated by the need for independent FPYs that reproduce cumulative fission yields and experimental data using the latest decay schemes, a comprehensive set of independent FPY for ${ }^{235} \mathrm{U}$ (thermal, fast), ${ }^{238} \mathrm{U}$ (fast), and ${ }^{239,241} \mathrm{Pu}$ (thermal) were developed using the England and Rider evaluations as a basis. The results were validated experimentally against measured cumulative FPYs in the particular case of krypton and xenon isotope ratios. The approach used in this work is based on a sequential Bayesian method that adjusts the independent fission yields using information from the cumulative yields to provide an internally consistent set of fission yield and nuclear decay data. Another important outcome of this approach is to generate covariance data on the FPYs that are necessary for fission product uncertainty analysis.

The revised FPY data and covariance matrices have been issued in the latest beta version 3 of the SCALE-6.2 code system for nuclear modeling and simulation. This code system includes the simulation codes that enable uncertainty analysis of spent nuclear fuel systems due to cross section, FPYs, and nuclear decay data uncertainties. These data are proposed as a revision and expansion to the ENDF/B-VII.1 nuclear data for fission yields.

\section{METHODOLOGY}

Research on FPY data and uncertainty evaluation methodologies is an emerging area of high interest to international organizations engaged in used fuel analysis. The Organization for Economic Co-operation and Development (OECD) under the Working Party on International Nuclear Data Evaluation Co-operation recently initiated a new sub-group 37 on FPY uncertainty methodologies (WPEC-37) 6]. Oak Ridge National Laboratory is currently working on developing methodologies to generate covariance matrices to enable fission product uncertainty quantification using fission yield data.

The methodology developed to adjust independent FPYs is based on a sequential Bayesian method developed by T. Kawano [3. However, unlike Kawano's method, the Bayesian method was applied not only on the long-lived or stable element(s) at the end of mass chain but also on each cumulative yield along the chain. This methodology allows the entire statistical information of the cumulative FPY data (central values and the available covariance information) to be included in the estimation of the related independent FPYs. In applying this method, the independent/cumulative FPYs no longer present in the ENDF/B-VII.1 decay schemes but still present in the FPY evaluations were eliminated. Most of these cases were related to metastable nuclei whose independent FPYs were added to the corresponding independent FPY in the ground state (e.g., ${ }^{84 \mathrm{~m}} \mathrm{As}$ and ${ }^{86 \mathrm{~m}} \mathrm{Br}$ ).

In defining the method, we start from the relation between cumulative and independent yields,

$$
C_{i}(\mathbf{I})=I_{i}+\sum_{j \in \mathbf{k}^{i}} C_{j}(\mathbf{I}) b_{i, j},
$$


where the index $i$ refers to each independent and cumulative yields identified by the triplet $(A, Z, M)$. Here, $A$ and $Z$ are the mass and charge number, respectively, while $M$ represents the isomeric state $(M=0$ for the ground state, $M=1,2, \ldots$ for the $1^{\text {st }}, 2^{\text {nd }}, \ldots$ meta states $)$. The index $j$ refers to all possible decay modes $\left(\beta^{-}, \beta\right.$-n, isomeric transition, ...) for the formation of the nucleus labeled by $i$. The index $j$ can be seen as running over the components of a vector $\mathbf{k}^{i}$ containing the pointers to specify the decaying nucleus identified by $j$ and the related fraction $b_{i j}$ of the precursor nucleus $j$ to the daughter nucleus $i$. The total number of nuclei decaying to the $i$-th nucleus is given by the dimension of vector $\mathbf{k}^{i}$, i.e., $n_{i}=\operatorname{dim}\left(\mathbf{k}^{i}\right)$. In matrix notation, the vector of the cumulative yields can be written as

$$
\mathbf{C}=\mathbf{S} \mathbf{I},
$$

where the vector I contains the independent FPYs ordered accordingly to the elements of the lower triangular matrix $\mathbf{S}$. In the simplest case where a set of nuclei in the ground state are decaying by $\beta^{-}$, the matrix elements of $\mathbf{S}$ can be written as

$$
s_{k l}=\prod_{j=l}^{k-1} b_{j+1, j} .
$$

Equation (2) can be seen as a system of coupled linear equations. The coupling among different equations depends on decay modes like $\beta$-n or $\alpha$-particle emissions, and the coefficients of the equations are defined by the branching ratios $b$. Therefore, the elements of the matrix $\mathbf{S}$ coincide with the partial derivatives of the $k$-th cumulative yield $C_{k}$ with respect to $l$-th independent yield as

$$
s_{k l}=\frac{\partial C_{k}(\mathbf{I})}{\partial I_{l}} .
$$

In the present work, the matrix $\mathbf{S}$ was computed on the basis of the ENDF-B/VII.1 decay sub-library and arranged according to the list of independent and cumulative FPY found in the ENDF-B/VII.1 library.

Here, we define the quantities relevant to the discussion of this work, using a notation similar to that found in Ref. 3. Using the evaluated cumulative yields $\zeta$ and related (diagonal) covariance matrix $\mathbf{Z}$ in the ENDF/BVII.1 library, one can obtain an updated set of values for independent yields $\mathbf{I}^{\prime}$ by

$$
\begin{aligned}
\mathbf{P}^{\prime} & =\mathbf{P}-\mathbf{P S}^{\mathrm{t}}\left(\mathbf{S P S}^{\mathrm{t}}+\mathbf{Z}\right)^{-1} \mathbf{S P}, \\
\mathbf{I}^{\prime} & =\mathbf{I}+\mathbf{P}^{\prime} \mathbf{S}^{\mathrm{t}} \mathbf{Z}^{-1}(\boldsymbol{\zeta}-\mathbf{C}(\mathbf{I})),
\end{aligned}
$$

where $\mathbf{P}$ is the covariance matrix (diagonal in this specific case) of $\mathbf{I}$ generated from ENDF/B-VII.1 uncertainties. In the sequential Bayesian update, the components of the vector $\mathbf{I}$ are considered as the set of parameters to compute the vector of the cumulative yields $\mathbf{C}(\mathbf{I})$ as defined in Eq.(1) and the decay scheme is the model to define the cumulative yields. The matrix $\mathbf{P}$ is the prior information on the independent FPYs, and additional correlations can be obtained from the model parameters of the independent yields. Here, we limit to consider that the independent FPYs, namely the components of the vector $\mathbf{I}^{\prime}$, need to satisfy three physical constraints, namely,

$$
\sum_{i} I_{i}^{\prime}=2, \quad \sum_{i} I_{i}^{\prime} A_{i}=A_{f}-\nu, \quad \sum_{i} I_{i}^{\prime} Z_{i}=Z_{f},
$$

where $A_{f}$ and $Z_{f}$ are the mass and charge number of the compound fissioning nucleus, respectively, and $\nu$ the number of prompt neutron emission. This can be achieved by similar equations, and for the normalization of the independent yields, one has

$$
\begin{aligned}
\mathbf{P}^{\prime \prime} & =\mathbf{P}^{\prime}-\mathbf{P}^{\prime} \mathbf{T}^{\mathrm{t}}\left(\mathbf{T} \mathbf{P}^{\prime} \mathbf{T}^{\mathrm{t}}+\sigma_{\mathrm{T}}^{2}\right)^{-1} \mathbf{T} \mathbf{P}^{\prime}, \\
\mathbf{I}^{\prime \prime} & =\mathbf{I}^{\prime}+\mathbf{P}^{\prime \prime} \mathbf{T}\left(2-\mathbf{T}^{\mathrm{t}} \mathbf{I}^{\prime}\right) / \sigma_{\mathrm{T}}^{2},
\end{aligned}
$$

where $\mathbf{T}$ is the unit vector and $\sigma_{\mathrm{T}}$ controls the precision of the integral over all fission products summing to 2 . For the mass and charge conservations, one has, respectively,

$$
\begin{aligned}
\mathbf{P}^{\prime \prime \prime} & =\mathbf{P}^{\prime \prime}-\mathbf{P}^{\prime \prime} \mathbf{U}^{\mathrm{t}}\left(\mathbf{U} \mathbf{P}^{\prime \prime} \mathbf{U}^{\mathrm{t}}+\sigma_{\mathrm{U}}^{2}\right)^{-1} \mathbf{U} \mathbf{P}^{\prime \prime}, \\
\mathbf{I}^{\prime \prime \prime} & =\mathbf{I}^{\prime \prime}+\mathbf{P}^{\prime \prime \prime} \mathbf{U}\left(A_{f}-\nu-\mathbf{U}^{\mathrm{t}} \mathbf{I}^{\prime \prime}\right) / \sigma_{\mathrm{U}}^{2},
\end{aligned}
$$

and

$$
\begin{aligned}
\mathbf{P}^{\prime \prime \prime \prime} & =\mathbf{P}^{\prime \prime \prime}-\mathbf{P}^{\prime \prime \prime} \mathbf{V}^{\mathrm{t}}\left(\mathbf{V} \mathbf{P}^{\prime \prime \prime} \mathbf{V}^{\mathrm{t}}+\sigma_{\mathrm{V}}^{2}\right)^{-1} \mathbf{V} \mathbf{P}^{\prime \prime \prime}, \\
\mathbf{I}^{\prime \prime \prime \prime} & =\mathbf{I}^{\prime \prime \prime}+\mathbf{P}^{\prime \prime \prime \prime} \mathbf{V}\left(Z_{f}-\mathbf{V}^{\mathrm{t}} \mathbf{I}^{\prime \prime \prime}\right) / \sigma_{\mathrm{V}}^{2},
\end{aligned}
$$

where the elements of the vector $\mathbf{U}$ and $\mathbf{V}$ are the $A$ and $Z$ numbers of the $i$-th fission product, i.e., $U_{i}=A_{i}$ and $V_{i}=Z_{i}$. Likewise in Eq. (6), $\sigma_{\mathrm{U}}$ and $\sigma_{\mathrm{V}}$ are used to control the precision of the integral, which, for the present work, was $10^{-5}$.

In this section we also describe a second way to define the Bayesian equations for the updated FPY data and related covariance matrix. This new formulation of the problem, practically, gives results identical to the sequential Bayesian update and has the advantage to include the constraints on the independent yields in a single set of equations. An updated covariance matrix $\mathbf{M}^{\prime}$ equivalent to that one obtained with the sequential Bayesian update can be defined by the relation

$$
\mathbf{M}^{\prime-1}=\mathbf{G}^{\mathrm{t}} \mathbf{M}^{-1} \mathbf{G},
$$

where $\mathbf{G}^{\mathrm{t}}=(\mathbb{I}, \mathbf{S}, \mathbf{T}, \mathbf{U}, \mathbf{V})^{\mathrm{t}}$ is the transpose rectangular matrix associated with the sensitivities of the prior parameters defined by the identity matrix $\mathbb{I}$ and the sensitivities of the cumulative FPY defined by $\mathbf{S}$. The other sensitivities $\mathbf{T}, \mathbf{U}, \mathbf{V}$ are for the three constrained parameters aforementioned. The elements of the diagonal matrix $\mathbf{M}$ are accordingly arranged as $\mathbf{P}, \mathbf{Z}$, and $\sigma_{\mathrm{T}}, \sigma_{\mathrm{U}}$, 
$\sigma_{\mathrm{V}}$. Due to the simple form of the matrix $\mathbf{M}$, the updated covariance matrix can be written in explicit form as

$$
\mathbf{M}^{\prime-1}=\mathbf{P}^{-1}+\mathbf{S}^{\mathrm{t}} \mathbf{Z}^{-1} \mathbf{S}+\mathbf{Q}^{-1}
$$

with

$$
\mathbf{Q}^{-1}=\frac{\left(\mathbf{T}^{\mathrm{t}} \otimes \mathbf{T}\right)}{\sigma_{\mathrm{T}}^{2}}+\frac{\left(\mathbf{U}^{\mathrm{t}} \otimes \mathbf{U}\right)}{\sigma_{\mathrm{U}}^{2}}+\frac{\left(\mathbf{V}^{\mathrm{t}} \otimes \mathbf{V}\right)}{\sigma_{\mathrm{V}}^{2}} .
$$

The updated set of parameters is given by

$$
\mathbf{I}^{\prime}=\mathbf{I}+\mathbf{M}^{\prime} \mathbf{S}^{\mathrm{t}} \mathbf{Z}^{-1}(\boldsymbol{\zeta}-\mathbf{C}(\mathbf{I})),
$$

where the contribution of the matrix $\mathbb{I}$ and vectors $\mathbf{T}, \mathbf{U}, \mathbf{V}$ is identically zero since we have chosen the vector of the prior parameters to coincide with the values found in ENDF/B-VII.1. One can notice that Eq. (11) differs from Eqs. (5.8) of the sequential method by the updated covariance matrix $\mathbf{M}^{\prime}$ where the uncertainties of the three physical constraints are included by the matrix Q.

\section{RESULTS}

The independent FPYs in ENDF/B-VII.1 and decay data were used by ORIGEN in order to simulate fission product concentrations for fission pulse, for instance, on ${ }^{235} \mathrm{U}$. The values of independent FPYs in the ENDF/BVII.1 sub-library were found to be inconsistent with the total mass chain cumulative FPY values and individual uncertainties in the ENDF/B-VII.1 library. The concentrations of key stable and long-lived isotopes were compared to concentrations in the current ENDF/B-VII.1 cumulative yield data. Figure 1 shows the ratios of the

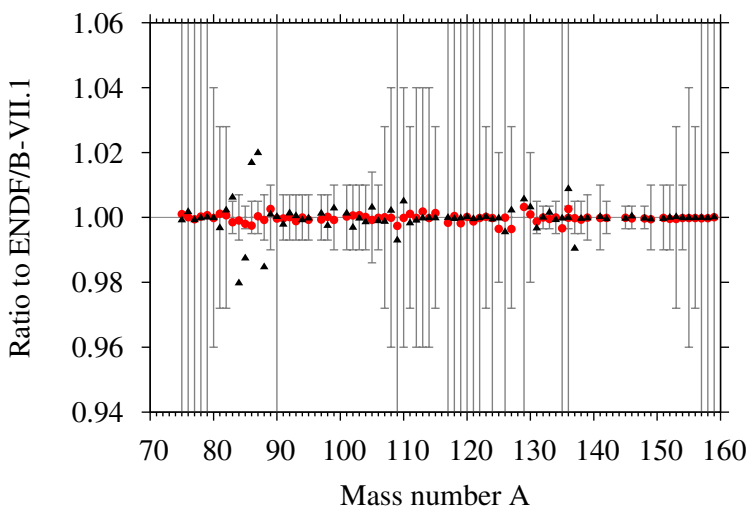

FIG. 1. (Color online) Ratios of the calculated (long-lived) cumulative yields of magnitude $>10^{-5}$ to ENDF/B-VII.1 library for $n+{ }^{235} \mathrm{U}$ at thermal energy. The cumulative FPYs were obtained by ORIGEN (cooling time $t=10^{16} \mathrm{~s}$ ) using the ENDF/B-VII.1 (triangles) and adjusted (dots) independent FPYs. The plotted relative uncertainties are directly taken from ENDF/B-VII.1 cumulative yields. long-lived cumulative yields calculated by ORIGEN using ENDF/B-VII.1 (triangles) and the adjusted (dots) independent yields to ENDF/B-VII.1 library for $n+{ }^{235} \mathrm{U}$ at thermal energy. Although the deviations for this particular set of nuclei is small, the ratios should be unitary in order to have consistency between independent and cumulative FPY data in ENDF/B-VII.1 library. The biggest deviations are seen in the mass region of $A=80$ 85. The major improvements of the adjusted FPYs are seen in this mass range, while the impact on short-lived nuclei due to the adjustment are still being investigated. For short-lived nuclei along the decaying chain, infact, discrepancies from ENDF/B-VII.1 cumulative yields are inevitable due to the changes in the branching ratios since the original evaluation of England and Rider. Motivated by these inconsistencies, independent FPYs and their covariance data for ${ }^{235,238} \mathrm{U}$ and ${ }^{239,241} \mathrm{Pu}$ in the thermal and fission spectrum neutron range were generated by a sequential Bayesian based on the ENDF/B-VII.1 decay sub-library. These specific actinides were chosen because they contribute to over $99 \%$ of all fissions in thermal reactors. Tests were performed in order to compare calculated and experimentally measured fission product noble gases, particularly, krypton and xenon isotopes. Detailed power histories were obtained and modeled using ORIGEN for three pressurized water reactors (Trino Vercellese, Obrigheim, and Vandellos Unit 2). These three reactors were chosen because of their spread in initial enrichment and final burnup. Isotopic composition measurements by mass spectrometry were determined for the krypton and xenon fission gases. Limited information on the uncertainties of these noble gas measurements was found. Experimental uncertainties for krypton and xenon isotopic ratios were reported (see Tab. II) for Trino Vercellese and Obrigheim facilities, but nothing was found for Vandellos facility.

TABLE II. The average relative standard deviations of the measurements of the fission gases isotopic ratios for Trino Vercellese [7] and Obrigheim [8] facilities.

\begin{tabular}{c|c||c|c}
\hline \hline \multicolumn{4}{c}{ Trino Vercellese facility } \\
\hline${ }^{83} \mathrm{Kr} /{ }^{86} \mathrm{Kr}$ & $0.3 \%$ & ${ }^{131} \mathrm{Xe} /{ }^{134} \mathrm{Xe}$ & $0.2 \%$ \\
${ }^{84} \mathrm{Kr} /{ }^{86} \mathrm{Kr}$ & $0.2 \%$ & ${ }^{132} \mathrm{Xe} /{ }^{134} \mathrm{Xe}$ & $0.2 \%$ \\
${ }^{84} \mathrm{Kr} /{ }^{83} \mathrm{Kr}$ & $0.3 \%$ & ${ }^{136} \mathrm{Xe} /{ }^{134} \mathrm{Xe}$ & $0.2 \%$ \\
- & - & ${ }^{132} \mathrm{Xe} /{ }^{131} \mathrm{Xe}$ & $0.2 \%$ \\
\hline \multicolumn{4}{c}{$\mathrm{Obrigheim}$ facility } \\
\hline${ }^{83} \mathrm{Kr} /{ }^{86} \mathrm{Kr}$ & $0.3 \%$ & ${ }^{130} \mathrm{Xe} /{ }^{134} \mathrm{Xe}$ & $0.5 \%$ \\
${ }^{84} \mathrm{Kr} /{ }^{86} \mathrm{Kr}$ & $0.2 \%$ & ${ }^{131} \mathrm{Xe} /{ }^{134} \mathrm{Xe}$ & $0.2 \%$ \\
${ }^{85} \mathrm{Kr} /{ }^{86} \mathrm{Kr}$ & $0.3 \%$ & ${ }^{132} \mathrm{Xe} /{ }^{134} \mathrm{Xe}$ & $0.2 \%$ \\
- & - & ${ }^{136} \mathrm{Xe} /{ }^{131} \mathrm{Xe}$ & $0.2 \%$ \\
\hline \hline
\end{tabular}

Figure 2 shows the measured ratios compared to the calculations performed using the ENDF/B-VII.1 library (full symbols, i.e., $\checkmark, \mathbf{\square}, \mathbf{\Delta}$ ) and the newly estimated inde- 
pendent FPYs (empty symbols, i.e., $\diamond, \square, \triangle)$. The super-
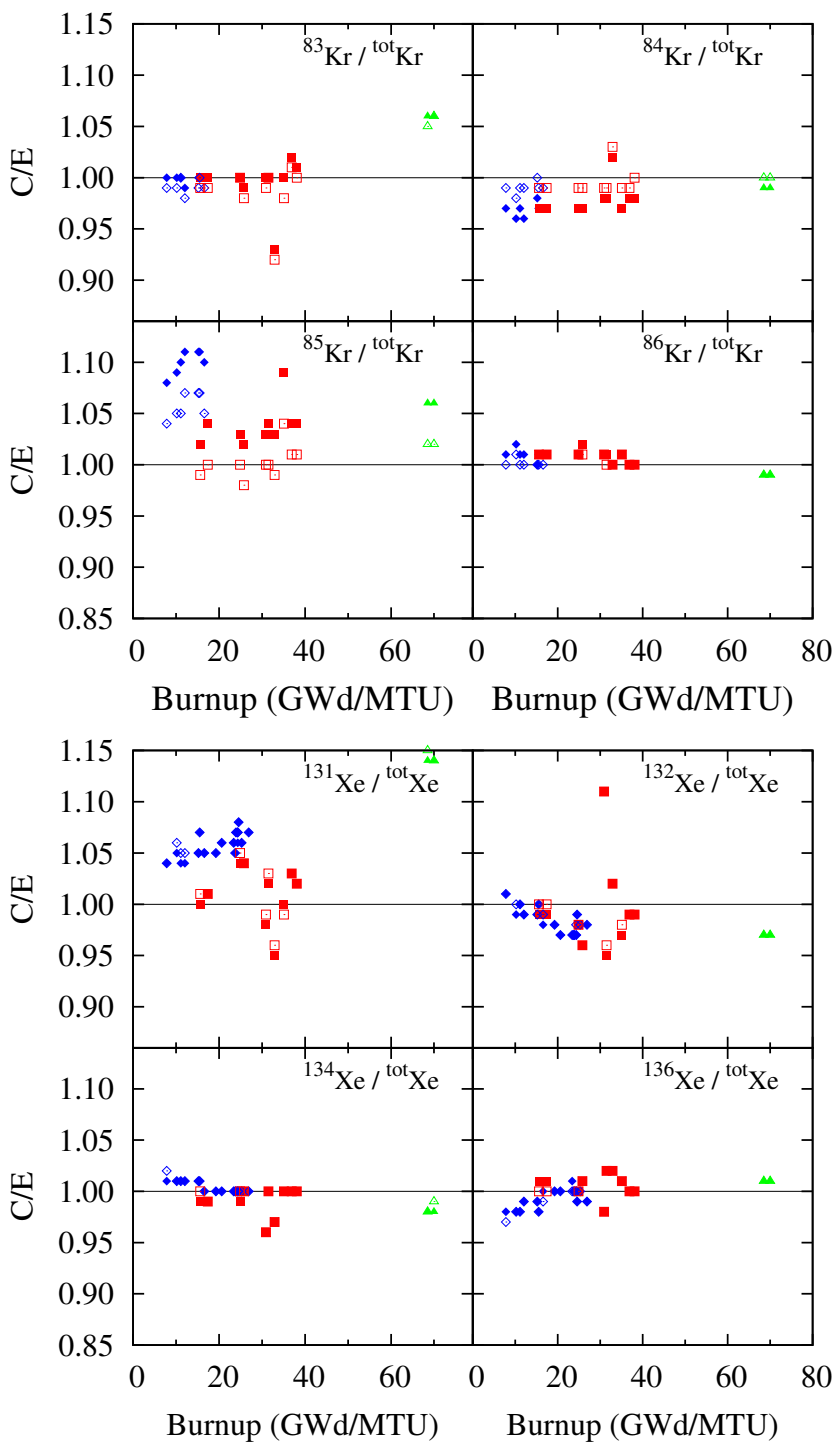

FIG. 2. (Color online) Ratio of calculated cumulative atom ratios (C) to experimentally measured ones (E) for krypton and xenon isotopes. Full symbols $(\boldsymbol{\nabla}, \mathbf{\square}, \mathbf{\Delta})$ refer to the ENDF/BVII.1 library and empty symbols $(\diamond, \square, \triangle)$ to the results obtained with improved independent FPYs. Different symbols refer to different experimental facilities: diamonds, Trino Vercellese [7]; squares, Obrigheim [8]; and triangles, Vandellos [9].

script "tot" refers to the sum of the four isotopes, while the different colors distinguish between different experimental facilities, namely, Trino Vercellese (diamonds) 7, Obrigheim (squares) [8, and Vandellos (triangles) [9]. In the case of krypton, major improvements are seen for ${ }^{84} \mathrm{Kr}$ and ${ }^{85} \mathrm{Kr}$, while minor ones for ${ }^{86} \mathrm{Kr}$. In the case of xenon isotopes, the two libraries performed on average very similarly, as shown in Fig. 2. The uncertainties of the plotted experimental data can be computed from the experimental uncertainties of Tab. II according to the simple linear error propagation that, e.g. for krypton isotopes, can be written as

$$
\Delta\left(\frac{{ }^{\mathrm{x}} \mathrm{Kr}}{{ }^{\mathrm{y} K r}}\right)^{2} \approx\left(\frac{{ }^{\mathrm{x}} \mathrm{Kr}}{{ }_{\mathrm{y}} \mathrm{Kr}}\right)^{2}\left[\left(\frac{\Delta^{\mathrm{x}} \mathrm{Kr}}{{ }^{\mathrm{x}} \mathrm{Kr}}\right)^{2}+\left(\frac{\Delta^{\mathrm{y} K r}}{{ }^{\mathrm{y} K r}}\right)^{2}\right],
$$

where we assumed the experimental data uncorrelated. It can be shown in the particular case of Trino Vercellese facility the plotted experimental ratios have relative standard deviations below $1 \%$.

Coupled with the results shown in Fig. 2, independent FPY covariance matrices (standard deviation and correlation) were also generated by the (sequential) Bayesian update. As an example we show in Fig. 3 the obtained

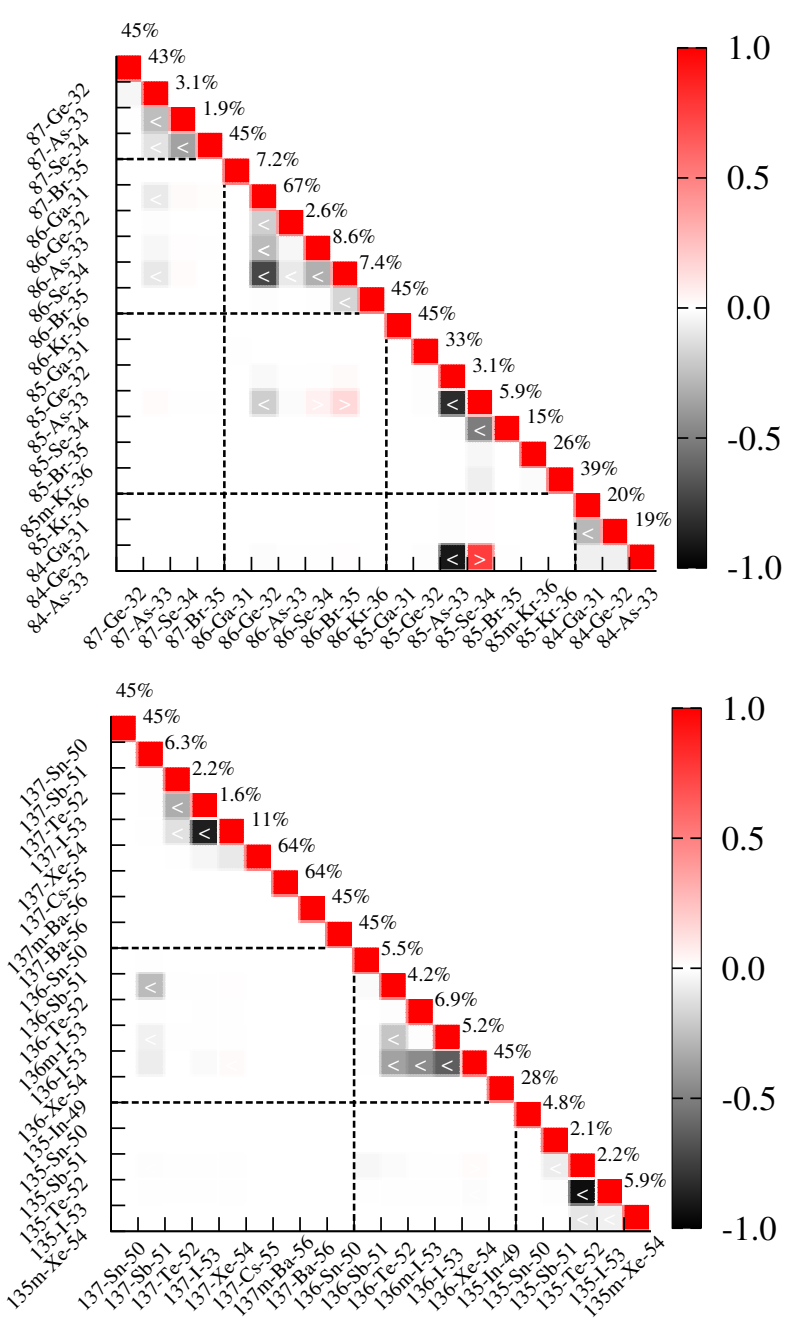

FIG. 3. (Color online) Lower triangular correlation matrices of $\mathrm{n}+{ }^{235} \mathrm{U}$ at thermal energy for the mass chains in the range of $\mathrm{A}=85-87$ and $\mathrm{A}=135-137$ obtained with the (sequential) Bayesian method. Non-negligible positive and negative correlations are highlighted by the symbols $>$ and $<$, respectively. The relative uncertainties are also shown along the diagonal. 
correlation matrices (in a lower triangular representation) and related uncertainties for krypton and xenon isotopes in the mass ranges of $A=85-87$ and $A=135-137$, respectively. Although both correlation matrices are very sparse, strong negative correlations are seen between independent yields with the same mass number; however, especially for krypton isotopes, one can also see nonnegligible positive correlations between elements of neighbouring masses that derive from the $\beta$-n emissions. These correlations and related uncertainties are of fundamental importance when the uncertainties of cumulative yields are calculated by error propagation from $\mathbf{I}$ to $\mathbf{C}$.

\section{CONCLUSIONS}

We have generated a revised set of independent FPYs and related covariance data for four actinides, i.e., ${ }^{235,238} \mathrm{U}$ and ${ }^{239,241} \mathrm{Pu}$ that provides greater consistency with cumulative yield values when used with modern ENDF/B-VII.1 decay data. These four actinides are particularly important for fission product analysis of spent nuclear fuel and, therefore, to validate the ORIGEN with experimentally measured cumulative FPYs for krypton and xenon isotopes, as shown in Fig. 2. The consistency of the independent and cumulative yields was achieved by 1) the use of the ENDF/B-VII.1 decay sub-library and 2) using cumulative yields and accounting for uncertainty information available from the England and Rider cumulative FPYs in the ENDF/B-VII.1 library. The first item was motivated to resolve the inability of ENDF/BVII.1 library to match the reported cumulative FPYs with those generated by the use of independent FPY and decay sub-libraries. The latter was necessary because the independent FPY uncertainties in the ENDF/B-VII.1 library appear incompatible with the FPY cumulative uncertainties.

The goal of the present work was to correct the inconsistencies within the ENDF/B-VII.1 library. Therefore, in the particular case of stable nuclei, we constrained our results to the cumulative FPY values in the library, giving them much smaller uncertainties.

Acknowledgments: This work was funded by the Defense Threat Reduction Agency Office of Nuclear Forensics Materials Collection, Analysis, Debris Diagnostics Branch, and the National Nuclear Security Administration, Office of Defense Nuclear Nonproliferation Research and Development.
[1] K.J. Moody et al., "Nuclear Forensic Analysis" (Taylor \& Francis Group, LCC 2005).

[2] M.B. Chadwick et al., "ENDF/B-VII.1 Nuclear Data for Science and Technology: Cross Sections, Covariances, Fission Product Yields and Decay Data," Nucl. DATA SheETs 112, 2887 (2011).

[3] T. Kawano et al., "Estimation of ${ }^{239} \mathrm{Pu}$ independent and cumulative fission product yields from the chain yield data using a Bayesian technique," J. Nucl. ScI. TeChnol. 50, 1034 (2013).

[4] T.R. England et al., "Evaluation and Compilation of Fission Product Yields 1993," Los Alamos National Laboratory Report LA-UR-94-3106, ENDF-349 (1994).

[5] S.M. Bowman, "SCALE-6: Comprehensive Nuclear Safety Analysis Code System," Nucl. Tech. 174, 126 (2011).

[6] Working Party on International Nuclear Data Evaluation
Co-operation (WPEC), Improved fission product yields evaluation methodologies - WPEC37, website http:// www. oecd-nea.org/science/wpec/sg37

[7] A.M. Bresesti et al., "Post-irradiation Analysis of Trino Vercellese Reactor Fuel Elements," Report EUR4909e (1972) and P. Barbero et al., "Post-irradiation Examination of the Fuel Discharged from the Trino Vercellese Reactor after the 2nd Irradiation Cycle," Report EUR5605e (1977).

[8] P. Barbero et al., "Post Irradiation Analysis of The Obrigheim PWR Spent Fuel," European Appl. Res. Report 2, 129 (1980).

[9] H.U. Zwicky et al., "Nuclide analysis in high burnup fuel samples irradiated in Vandellos 2," J. NuCL. MATER. 402, 60 (2010). 